\title{
Management of Traumatic Soft Tissue Injuries of the Face
}

\author{
Daniel Y. Cho, MD, PhD ${ }^{1} \quad$ Brooke E. Willborg ${ }^{2} \quad$ G. Nina Lu, MD 3 \\ ${ }^{1}$ Division of Plastic Surgery, Harborview Medical Center, University of \\ Washington, Seattle, Washington \\ 2 Elson S. Floyd College of Medicine, Washington State University, \\ Spokane, Washington \\ ${ }^{3}$ Department of Otolaryngology-Head and Neck Surgery, Harborview \\ Medical Center, University of Washington, Seattle, Washington \\ Address for correspondence G. Nina Lu, MD, Harborview Medical \\ Center, 325 9th Avenue, 4 West, Seattle, WA 98104 \\ (e-mail: ninalu@uw.edu).
}

Semin Plast Surg 2021;35:229-237.

\begin{abstract}
Keywords

- facial trauma

- soft tissue injury

- laceration

- facial nerve

- parotid duct

Facial soft tissue injuries encompass a broad spectrum of presentations and often present significant challenges to the craniofacial surgeon. A thorough and systematic approach to these patients is critical to ensure that the patient is stabilized, other injuries identified, and the full extent of the injuries are assessed. Initial management focuses on wound cleaning with irrigation, hemostasis, and debridement of nonviable tissue. Definitive management is dependent on the region of the face involved with special considerations for critical structures such as the globe, lacrimal apparatus, facial nerve, and parotid duct. Following sound surgical principles, these injuries can be managed to maximize both functional and aesthetic outcomes while minimizing complications.
\end{abstract}

Soft tissue injuries of the face are commonly encountered by providers caring for trauma patients with lacerations of the face and scalp accounting for $\sim 50 \%$ of the wounds treated in the emergency department. ${ }^{1}$ These injuries can be isolated to the soft tissues or be associated with craniofacial fractures. The spectrum of facial soft tissue injuries is broad, ranging from straightforward lacerations requiring simple repairs to complex injuries requiring multidisciplinary care. The significant variability in presentation can make the management of soft tissue injuries of the face both mundane and challenging. Regardless of the injury, facial soft tissue injuries require careful assessment and management to ensure optimal aesthetic and functional outcomes. $^{2}$ The goal of this work is to share some of the pearls and pitfalls in the management of soft tissue trauma of the face based on our experience at a regional level 1 trauma center.

\section{Initial Evaluation}

\section{Primary Survey}

The initial presentation of soft tissue injuries can vary significantly in terms of severity, complexity, and concomitant injuries. The initial assessment should follow wellestablished, systematic trauma protocols such as the Advanced Trauma Life Support (ATLS) program developed by the American College of Surgeons (ACS) Committee on Trauma (CoT) for the accurate and rapid assessment of a patient's condition and injuries, resuscitation, and transfer to higher levels of care if necessary. ${ }^{3,4}$ The primary survey focuses on airway evaluation with cervical spine control, breathing with adequate oxygenation and ventilation, circulation to ensure adequate tissue perfusion, disability, and exposure of all injuries. ${ }^{4-6}$ At our center, this is a multidisciplinary approach involving emergency medicine, trauma
Issue Theme The Harborview Approach to Craniofacial Trauma; Guest Editor: Craig Birgfeld, MD, FACS (c) 2021. Thieme. All rights reserved. Thieme Medical Publishers, Inc., 333 Seventh Avenue, 18th Floor, New York, NY 10001, USA
DOI https://doi.org/ 10.1055/s-0041-1735814. ISSN 1535-2188. 
surgery, anesthesia, and the craniofacial service. Facial soft tissue injuries can be associated with threats to the airway requiring airway stabilizing maneuvers or devices, intubation, or surgical airway. ${ }^{5}$ Injuries of the head and neck region can be associated with life-threatening blood loss and ensuring appropriate hemostasis and resuscitation is necessary. ${ }^{6}$

\section{Secondary Survey}

Once the patient is stabilized, the secondary survey ensues with a thorough head-to-toe physical examination to identify all anatomic injuries. This exam is informed by a thorough history including detailed information about the mechanism of the injury, symptoms, allergies, medications, past medical and surgical history, and last per os (PO) intake. $^{7}$ Based on the secondary survey, imaging studies including plain film radiographs and/or cross-sectional imaging are obtained to better elucidate specific injuries. Other considerations include a tetanus vaccine booster and antibiotic prophylaxis for bite injuries or gross contamination.

\section{Craniofacial Exam and Imaging}

A complete head and neck exam should be performed by the craniofacial surgery team. This includes an assessment of all soft tissue injuries, cranial nerve exam, and an evaluation for bony trauma. A more thorough discussion is included below by anatomic region. Standard imaging at our center includes a maxillofacial computed tomography (CT) scan with 3dimensional reconstructions to identify bony injuries. ${ }^{8,9}$ Plain films in craniofacial injuries are not preferred due to their limited diagnostic accuracy and utility for surgical planning. While a dental panoramic radiograph is useful for evaluating the upper and lower jaw, this is not the optimal imaging modality in the trauma setting. ${ }^{9} \mathrm{CT}$ angiography of the head and neck are obtained for patients at high risk for or with symptoms of blunt cerebrovascular injury (BCVI) (-Table 1). ${ }^{10-12}$

Table 1 Considerations for CT angiography of the head and neck to evaluate for blunt cerebrovascular injury

\begin{tabular}{|l|}
\hline High risk factors for BCVI \\
\hline Lefort II or III Fracture \\
Mandible fracture with high energy mechanism \\
All skull base fractures \\
Any fracture of C1, C2, or C3 \\
Traumatic cervical spine subluxation \\
Cervical spine fracture extending to transverse foramen \\
Cervical spine fracture with thoracic or lumbar spine \\
fractures \\
Great vessel injury in the thorax \\
\hline Signs or Symptoms of BCVI \\
\hline Arterial hemorrhage from the neck, mouth, nose, or ears \\
Gunshot wounds to the head, face, or neck \\
Near hanging resulting in cerebral anoxia \\
Large or expanding cervical hematoma \\
Carotid Bruit in a patient $<50$ years old \\
Cerebral infarction on CT or MRI \\
Unexplained central or lateralizing neurologic deficit, TIA, \\
or Horner's syndrome \\
Diffuse axonal injury with GCS $<6$ \\
\hline
\end{tabular}

\section{Principles of Early Management}

\section{Irrigation and Debridement}

Initial management focuses on wound cleaning with irrigation, hemostasis, and debridement of nonviable tissue. Patient comfort may be improved with local or regional anesthesia prior to management. Irrigation with normal saline is recommended, with the use of syringe or pulse lavage for increased pressure when needed. ${ }^{13}$ In cases of gross contamination, the use of a dilute soap solution for irrigation can be considered. Antiseptic solutions such as chlorhexidine and povidone are not routinely used due to the risk of cellular injury to the traumatized tissue and mucosal surfaces. ${ }^{13}$ At our center, in small wounds without gross contamination, we recommend large volume irrigation ( $500 \mathrm{cc}$ to $1 \mathrm{~L}$ of normal saline) to appropriately cleanse the wound. In larger or grossly contaminated wounds, large volume irrigations should continue until the wound appears clean without any evidence of contamination. Manual extraction of embedded foreign material may be necessary to fully decontaminate the wound. Hemostasis is initially controlled with direct pressure and/or the addition of epinephrine to local anesthetic. Electrocautery of injured vessels should be reserved for uncontrollable bleeding, to spare additional damage to surrounding soft tissue. Arterial bleeding may accompany cranial nerve transection (such as the facial or trigeminal nerves) and targeted hemostasis helps prevent further damage to traumatized nerve endings. Debridement of obviously nonviable tissue should be completed conservatively to preserve adequate tissue for repair. When in doubt, the practitioner should err on the side of tissue preservation. Given the significant vascularity of the face, repaired tissue may survive or engraft following repair. ${ }^{13}$

\section{Soft Tissue Abrasions and Traumatic Tattoos}

Soft tissue abrasions that spare full thickness injury to the dermis do not require surgical closure but should follow the principles of wound irrigation and debridement. Soft tissue lacerations disrupting the dermis should ideally undergo primary closure within 8 hours of the injury. ${ }^{2}$ Partial thickness abrasions may be treated conservatively with local wound care. ${ }^{2}$ We typically recommend topical petroleum ointment or $3 \%$ bismuth tribromophenate in a petroleum based fine mesh gauze (Xeroform) for initial wound care as it is effective and simple for patients to learn in the acute setting. Patients should be counseled that bacitracin ointment can cause a contact dermatitis in $15 \%$ of patients and if used, should be limited to 3-4 days duration. ${ }^{14}$ Blast, ballistic, firework, and road rash injuries can all result in particulate inclusions producing a traumatic tattoo (-Fig. 1). ${ }^{15}$ Early management starts with scrubbing the wound using sponges, surgical scrub brushes, or wire brushes within 6-72 hours, followed by forceps removal of particles by surgical loupe vision. Secondary management of long-lasting traumatic tattooing includes dermabrasion direct excision, or laser treatment. Laser treatment is not recommended for gunpowder injuries due to the risk of igniting the inclusions and producing significant tissue damage. $^{16}$ 


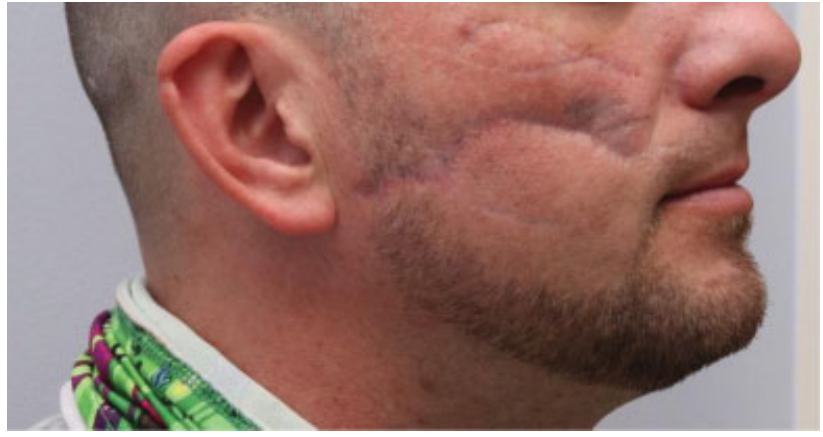

Fig. 1 Traumatic tattooing of the right cheek from embedding of particulate matter (in this case asphalt) into an open soft tissue injury.

\section{Soft Tissue Avulsions}

Avulsion injuries are characterized by the separation and subsequent loss of tissue. ${ }^{2}$ Varying degrees of soft tissue loss may be involved with marginal, partial, or complete necrosis. In the acute setting, the avulsed tissue should be assessed for viability. This involves evaluating the dimensions of the intact skin bridge as well as visual inspection of the tissue including capillary refill of the avulsion flap and bleeding from the wound edges. Tissue that is obviously non-viable should be debrided conservatively. Otherwise, primary repair should be attempted. Elasticity and edema of avulsed tissue may cause soft tissue retraction, placing undue tension on the flap with anatomic repair. In these cases, the tissue should be laid down on the wound with minimal tension and gently sutured in that position to prevent necrosis of the entire avulsion flap. The remaining open areas of the wound should be allowed to heal secondarily with local wound care. Amputation of specialized tissue such as the auricle, nose, and lip may be amenable to microvascular replantation if an identifiable artery is available for anastomosis. If venous anastomosis is not available, hirudotherapy, also known as medicinal leach therapy, may be used to ease venous congestion until neovascularization occurs. Significant soft tissue loss not amenable to microvascular replantation may require delayed local rotational flaps, grafts, or free tissue transfer. ${ }^{17}$

\section{High Energy Injuries}

High energy injuries such as ballistic or firework injuries often require complex multidisciplinary care. In the acute phase, establishing a secure airway and enteral access is important in initial resuscitation. Placement of tracheostomy and gastrostomy tube may also facilitate future reconstructive needs. ${ }^{4,5}$ Urgent irrigation and debridement, preliminary skeletal fixation, and soft tissue repair is recommended as it has been shown to reduce the number of surgical procedures, shorten hospitalizations, and reduce rates of soft-tissue contracture. ${ }^{18}$ Once the patient is physiologically stable, definitive reconstruction can be performed in a staged fashion. It is important to consider the psychosocial impacts of the injury and recovery process for the patient and provide adequate counseling and support.

\section{Evaluation and Management by Anatomic Region}

\section{Scalp}

\section{Evaluation and Special Considerations}

A careful examination of the scalp must be performed in patients with facial trauma as lacerations can be hidden by the hair. Injuries to the scalp are associated with heavy bleeding due to the rich vascular supply of the region. Hemostasis is critical for limiting blood loss and improving visualization for repair. Electrocautery should be used precisely to limit injury to hair follicles and prevent alopecia ${ }^{2}$; direct pressure is effective but careful ligation of injured vessels may be required. Routinely shaving the scalp prior to repair is not necessary and can be associated with an increased risk of infection. ${ }^{19}$ However, in some cases removing surrounding matted hair may be necessary for sufficient wound cleansing and visualization. Care should be taken to prevent stray hair from entering the wound. ${ }^{12}$ Using a local anesthetic containing epinephrine can help with hemostasis to facilitate repair. The addition of $8.4 \%$ bicarbonate solution to $1-2 \%$ lidocaine in a $1: 10$ ratio improves discomfort associated with injection of local anesthetic by neutralizing the acidity of lidocaine.

\section{Management}

The goals of managing scalp injuries are to achieve a tensionfree closure, restore the structure of the scalp, and protect the cranium. ${ }^{2}$ Lacerations can usually be repaired with primary closure ${ }^{20}$; a layered repair is recommended including periosteum, galea, and dermis. Undermining tissue or scoring the galea can be considered to facilitate the closure in areas of high tension. Surgical staples are preferred for superficial layer of closure in scalp lacerations. Injuries with large areas of dead space may require placement of a drain and a pressure wrap to reduce the risk of hematoma, seroma, and secondary complications. In cases of complete scalp avulsion, replantation should be considered if the scalp has been preserved appropriately and microsurgical capabilities are available. ${ }^{21}$

\section{Forehead}

\section{Evaluation and Special Considerations}

Initial examination of the forehead includes an evaluation of the facial musculature. The frontal branch of the facial nerve innervates the frontalis, orbicularis oculi, and corrugator supercilli muscles. This nerve can be found along Pitanguy's Line, which is defined as a line connecting $0.5 \mathrm{~cm}$ below the tragus to $1.5 \mathrm{~cm}$ above the lateral eyebrow. ${ }^{22}$ For any injuries in this area, the function of this nerve must be assessed and documented prior to injecting local anesthetic or attempting repair. ${ }^{22}$ The sensory innervation of the forehead is derived from the supraorbital and supratrochlear nerves, which can be blocked directly via injection of local anesthetic along the medial third of the supraorbital rim. ${ }^{23}$ The addition of 
epinephrine and bicarbonate to local anesthetics can help with hemostasis and enhance comfort, respectively. ${ }^{24}$

\section{Management}

Management should focus on reapproximating the soft tissue with careful consideration to the brow margins, existing forehead rhytids, and hairline of the forehead. Primary repair is often possible, particularly without substantial tissue loss. Undermining the surrounding tissue can relieve tension. Smaller defects may benefit from elevation of a subcutaneous flap while larger defects may benefit from elevation of a subgaleal flap. ${ }^{25}$ The level of tissue elevation may also be determined by the depth of the injury or avulsion of existing tissue. Relaxing incisions in the galeal (also known as galeotomies) perpendicular to the vector of maximal tension allows for additional tissue advancement. While staples are appropriate on the scalp, they should be avoided on facial skin due to the associated risk of scar formation at the penetration site. ${ }^{25}$ If the eyebrows are involved, they should not be shaved but rather used as important landmarks for repair. ${ }^{19}$ If additional skin incisions are necessary to facilitate closure, designing the incision in the horizontally oriented relaxed skin tension lines is preferred. ${ }^{25}$ Healing by secondary intention can result in adequate cosmesis and secondary scar revision could result in a lower overall scar burden than the use of local flaps or skin grafts in the acute setting.

\section{Periorbita and Eyelids}

\section{Evaluation and Special Considerations}

Trauma to the periorbital region should raise concern for associated orbital or globe injury. It is important to perform a thorough examination including visual acuity, extra-ocular movements, intraocular pressure (IOP) measurements, and fluorescein eye stain test. ${ }^{26}$ Additionally, physical examination should include an assessment for the presence of bony step-offs, enophthalmos, or vertical dystopia, which are suggestive of orbital bone injury. ${ }^{26}$ Telecanthus suggests an underlying bony injury or avulsion of the medial canthal tendon. ${ }^{27}$ Any evidence of fat herniation into the laceration should raise concern for violation of the orbital septum, which necessitates operative exploration. Lacerations near the middle third of the eye should raise concern for a lacrimal apparatus injury, especially when associated with epiphora, which will require interrogation of the canaliculi. ${ }^{28}$ Early ophthalmology consultation is recommended for globe or lacrimal apparatus injury.

Severe orbital pain, difficulty opening the eyes, proptosis, or loss of vision with elevated IOPs are suggesting of retrobulbar hematoma or orbital compartment syndrome. ${ }^{4,7}$ This is a vision-threatening emergency and requires immediate lateral canthotomy and cantholysis for decompression; patients should also be counseled that this could develop in a delayed fashion and be given strict return precautions.

The innervation of the eyelids is complex and includes territories of the supratrochlear, supraorbital, lacrimal, zygomaticofacial, infraorbital, and infratrochlear nerves. ${ }^{23}$ While specific nerve blocks could allow for anesthesia without affecting the motor function of the orbicularis oculi muscle, local infiltration is typically necessary for complete anesthesia.

\section{Management}

As discussed above, the management of periorbital injuries may require multidisciplinary care between craniofacial surgery and ophthalmology; in some hospital systems, oculoplastic subspecialists may primarily manage these injuries. Once again, primary repair is preferred when possible. Eyelid anatomy is divided into anterior lamella (skin and orbicularis oculi) and posterior lamella (tarsus and conjunctiva). The surgeon should identify the level of injury and repair the lamella individually. ${ }^{27}$ Care must be taken not to injure the globe, obstruct the lacrimal apparatus, or cause a retrobulbar hematoma. Sutures should be placed at the gray line and lash line in an everted fashion to restore the contour of the eyelid margin followed by repair of the tarsal plate with resorbable sutures. When repairing the anterior lamella, care should be taken to only incorporate skin into the suture. Reapproximating the orbicularis oculi muscle is not necessary and the incorporation of deeper tissues in the repair can cause scarring of the eyelid skin to the orbital septum, cause tethering of the eyelid (-Fig. 2). Small injuries to the conjunctiva can heal secondarily while larger injuries require repair with a fine resorbable suture to prevent entropion. Injuries with less than $25 \%$ full thickness loss can be closed primarily $^{27}$ while larger defects require more complex reconstructions such as lid-switch flaps, Tenzel semicircular advancement flaps, and grafting. ${ }^{29}$ Any shortening or deficiency in the lamella as well as scarring can result in entropion or ectropion; scar massage and observation can often result in resolution of ectropion over time while shortening of the lamella and severe scarring will require secondary grafting.

Cannalicular injuries require interrogation by an ophthalmologist or other provider experienced in treating these injuries. The canaliculi appear as thin white tubular structures in the medial third of the eyelids and are better visualized under loupe magnification. Once injuries are identified, they are usually repaired over a silastic lacrimal stent, which is left in place for $2-3$ months. ${ }^{28}$

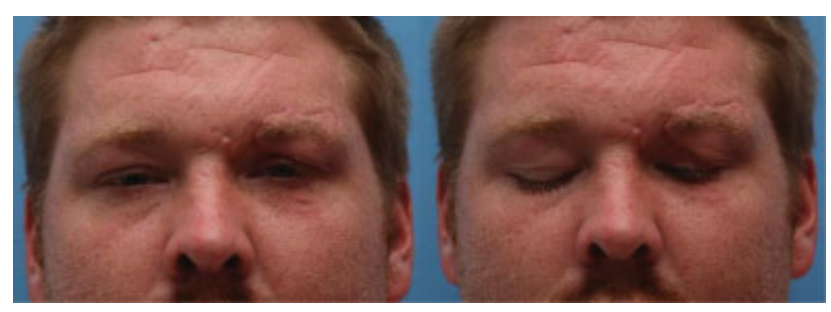

Fig. 2 Upper lid Lagophthalmos following left upper eyelid laceration repair with deepening of left upper eyelid fold with the eye open (left) and medial lagophthalmos with eye closure (right) due to tethering of the upper eyelid to the orbital septum. 


\section{Ears}

\section{Evaluation and Special Considerations}

The ears are unique that the anterior skin is tightly adherent to the underlying auricular fibrocartilage that gives shape to the external ear while the posterior skin is thicker and more mobile with a less complex topography. ${ }^{30}$ They are prone to injury because they are thin, exposed on two sides, and prominent. Visual examination of the ears is usually sufficient for diagnosis and treatment. Any skin loss or damage to the underlying cartilage should be identified. The external auditory canal (EAC) also should be examined with an otoscope to evaluate for foreign bodies, hemotympanum, tympanic membrane perforation, and lacerations. Skull base fractures can be associated with mastoid ecchymoses (Battle sign), ${ }^{31}$ CSF otorrhea, or otorrhagia. Lacerations of the anterior EAC may be associated with mandible fractures due to close proximity with the temporomandibular joint. It is important to ensure that the patient does not have an auricular hematoma, a subperichondrial collection of blood due to shear forces that separate the attachments of the soft tissue to the perichondrium. ${ }^{30,32}$ This can take hours to develop following blunt trauma and presents as a painful swelling that obliterates the normal topography of the ear. If untreated, the blood will clot and organize into a fibrotic mass that can calcify and result in a deformity known as a "cauliflower ear"32.

The sensory innervation of the ear is also complex. The upper two-thirds of the anterior surface is innervated by the auriculotemporal nerve and the posterior surface is innervated by the lesser occipital nerve. The great auricular nerve provides sensation to the lower third of the ear. ${ }^{23}$ These nerves can be blocked by injecting local anesthetic in a diamond pattern around the ear. ${ }^{33}$ However, the concha and EAC are innervated by the auricular branch of the vagus nerve, Arnold's nerve, which requires direct infiltration for anesthesia.

\section{Management}

The majority of ear lacerations can be repaired primarily. The blood supply to the ear is robust and the cartilage will survive if one surface remains in contact with well vascularized tissue. Sutures should be placed to approximate the skin at known anatomic landmarks to prevent distortion of the ear then the rest of the skin repaired. The cartilage does not need to be routinely repaired if the soft tissue envelope repair restores the normal contours. ${ }^{34}$ Soft tissue injuries around the EAC can result in stenosis or occlusion of the canal so stenting with an ear wick and instillation of antibiotic ear drops such as ciprofloxacin may be necessary. ${ }^{35}$ The data are unclear on the use of antibiotics to prevent chondritis and routine use is not indicated. Some authors recommend antibiotic use in the setting of grossly contaminated, degloved or exposed cartilage. Efforts should be made with local advancement of the soft tissues to provide coverage for the entirety of the cartilage but if some remain exposed, local wound care can be performed with mafenide acetate, which has good penetration into the cartilage

Amputations of the ear provide unique challenges with vascularity. Incomplete amputations should be primarily repaired, and success has been reported even in millimeter thin pedicle remnants. Those with marginal pedicles may have inadequate venous outflow so the ear should be monitored following repair for the early initiation of hirudotherapy/medicinal leech therapy if there is evidence of venous congestion. For complete amputations, microsurgical replantation can be considered whenever feasible as this will result in the best cosmesis if there is an adequate vascular pedicle. ${ }^{36}$ Case Reports have shown survival after replantation based on a single artery with the use of leeches for venous outflow. ${ }^{37}$ For complete amputations of partial ears, there are many reported strategies including subcutaneous banking of the cartilage or coverage with local and regional flaps. However, the denuded cartilage does not retain its shape and often becomes fibrotic and shapeless, resulting in a suboptimal outcome. Therefore, our center usually recommends local advancement of the skin for primary closure and staged secondary reconstruction (-Fig. 3). ${ }^{36}$ Preserving the postauricular skin and blood supply to temporoparietal fascia during primary repair aids in secondary reconstructive options.

The management of auricular hematomas requires drainage of the hematoma, hemostasis, and application of a

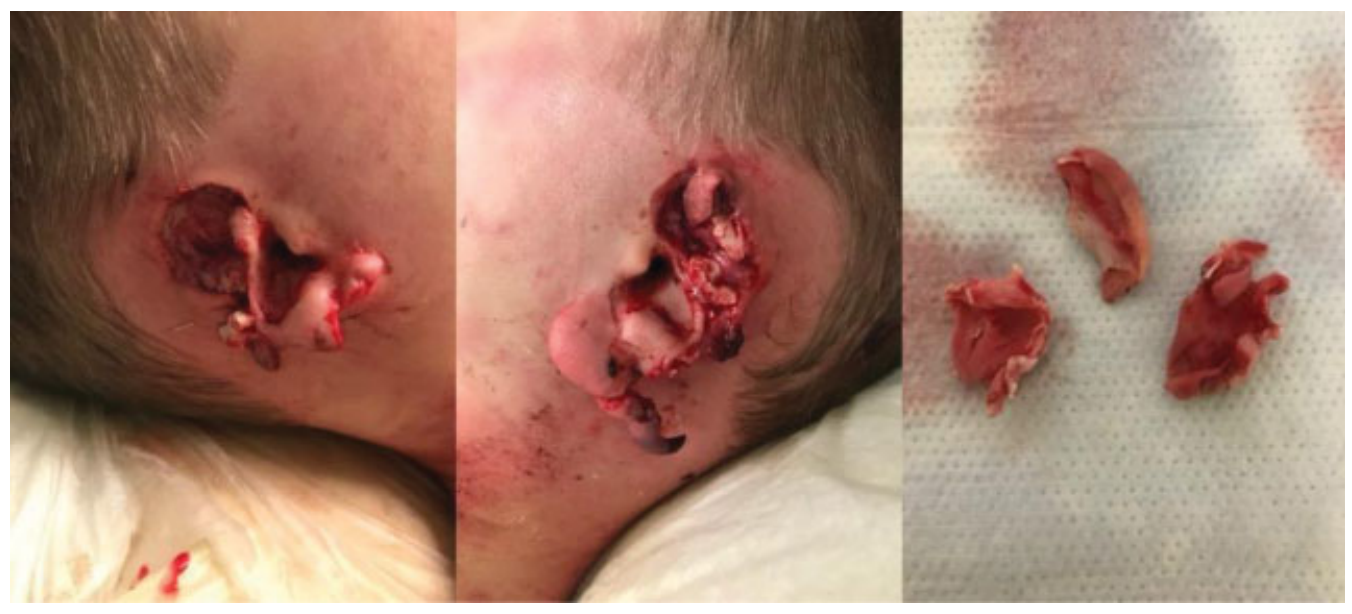

Fig. 3 A significant soft tissue and cartilage amputation from bilateral ears following a dog attack (left, center). The amputated parts were available (right) but given the mechanism and extent of the injury with no identifiable blood vessels for replantation, the decision was made to stabilize the soft tissues and plan for a staged reconstruction. 
bolster to prevent reaccumulation of the blood. ${ }^{32}$ Simple aspiration is insufficient since it will remove the hematoma but not prevent the reaccumulation into the subperichondrial space. Drainage can be accomplished through an incision over the hematoma, typically parallel to the antihelix or helix. The skin and perichondrial flap are elevated and suction is used to remove the hematoma. After irrigation, hemostasis must be achieved. At our center, we form a bolster using Xeroform and/or dental rolls, mold it to the contours of the ear, and secure it in place with through-andthrough sutures. A headwrap is applied and the bolsters are removed in 5-7 days. ${ }^{29}$

\section{Nose}

\section{Evaluation and Special Considerations}

The prominence of the nose on the central face predisposes it to injury. Nasal bone fractures are the most common facial fractures and usually occur without any soft tissue injuries. The nose consists of three components including the external covering, support structures, and nasal lining. Examination of the nose involves an assessment of the soft tissues and bony/cartilaginous framework as well as the external and internal anatomy. Any external lacerations and tissue loss as well as asymmetries and deviations of the nasal dorsum should be identified and associated with any palpable fractures or crepitus of the nasal bones and cartilages. An internal nasal exam should be performed with a nasal speculum, adequate light, and suction to evaluate for bleeding, mucosal lacerations, exposed cartilage or bone, septal injuries, and septal hematoma. ${ }^{38}$ Airflow through the nose should be assessed and the patient should be asked about previous nasal injuries and surgeries as well as breathing problems.

Nasal hemorrhage can cause significant blood loss, distress, and airway compromise. ${ }^{4,6,39}$ Initial management includes head of bed elevation, external pressure, application of nasal decongestant, and placement of nasal packing to apply direct pressure on the nasal mucosa for hemostasis. ${ }^{39}$ A variety of strategies exist for nasal packing and to achieve hemostasis, including the use of vasoconstrictive agents including oxymetazoline, phenylephrine, or cocaine and hemostatic agents such as topical tranexamic acid, oxidized cellulose polymer (Surgicel), and fibrin sealant (Tisseal). ${ }^{40}$ Direct nasoendoscopy may be required for targeted cautery, particularly in the posterior nasal cavity. If there is continued hemorrhage with placement of anterior nasal packing, posterior nasal packing can be performed with the passage of a Foley catheter through the nares, inflation of the balloons, and pulling back of the cathethers to apply pressure to the posterior mucosa. ${ }^{4}$ Placement of a posterior pack requires continuous hemodynamic monitoring in an intensive care unit. If hemorrhage is uncontrollable, either IR embolization or surgical exploration may be necessary. ${ }^{39}$

Nasal septal hematomas appear as a central swelling of the septal mucosa often causing complete occlusion of bilateral nasal cavities. These occur from blood accumulation between the perichondrium and the septal cartilage. This results in cartilage necrosis and must be identified and managed acutely to prevent the development of septal perforation, septal abscess, and saddle nose deformity. ${ }^{38}$

Given the complex innervation of the nose, multiple injections of local anesthetic are required for a combination of nerve blocks and local infiltration. The infraorbital nerve blocks can provide anesthesia to the lateral nasal side walls. ${ }^{41} \mathrm{~A}$ nasal block can be performed with the injection of anesthetic along the superior, lateral, and inferior aspect of the nose to numb the nasal dorsum, side wall, and tip. ${ }^{23}$ However, contributions from the anterior ethmoid nerve is difficult to inject intranasally with local anesthetic. Mucosal anesthesia can be achieved with nasal packing using cottonoids soaked in an anesthetic solution. ${ }^{41}$ Major nasal injuries may benefit from general anesthesia.

\section{Management}

The goal of nasal repair is to restore normal appearance without secondary nasal obstruction. Lacerations should be repaired in layers from the inside out. The nasal lining is repaired first with resorbable sutures with the goal of prevent intranasal cartilage exposure. Lacerations of the upper and lower lateral cartilages should be repaired to restore the structural framework of the nose. When repairing the skin, sutures should be placed to align the nasal landmarks to ensure an appropriate shape and contour of the nose. In particular, eversion of skin along the nostril margin may help prevent future notching from scar contracture. In general, loss of nasal soft tissue should be treated with local flaps and grafting within weeks of the injury as there is significant potential for contracture and collapse with secondary healing. ${ }^{42}$ Severe injuries often require delayed nasal reconstruction or rhinoplasty, ${ }^{42}$ which can make future reconstruction and rhinoplasty challenging.

If a nasal septal hematoma is identified, it should be evacuated to prevent long term complications and deformities. ${ }^{38} \mathrm{~A}$ small incision is made at the caudal aspect of the hematoma, which is then drained using suction and irrigated with saline. Once this is complete, hemostasis is ensured and the perichondrium is replaced over the septum. Multiple through-andthrough resorbable sutures are placed through the septum to bolster the perichondrium and prevent reaccumulation of blood. Doyle splints or nasal packing may be applied for additional pressure to prevent reaccumulation of blood.

\section{Cheek}

\section{Evaluation and Special Considerations}

Evaluation of cheek lacerations requires a thorough assessment of facial nerve and parotid duct injury as these must be identified and addressed immediately.

The facial nerve exits the skull base through the stylomastoid foramen and divides into its five main branches within the parotid gland. ${ }^{43}$ The zygomatic and buccal branches are at the highest risk for injury with cheek lacerations due to their location. ${ }^{44}$ The zygomatic branch runs inferior to the zygomatic arch while the buccal branch travels over the masseter muscle parallel to the parotid duct. Multiple branches and communicating branches exist, 
particularly medial to a vertical line from the lateral canthus. Injury to zygomaticobuccal branches medial to the lateral canthus in the midface do not typically require nerve exploration due to expected redundancy and compensation. A thorough examination of facial nerve function should be performed prior to injection of local anesthetic to assess for signs of facial paralysis.

The parotid duct, also known as Stenson's duct, follows a line from the tragus to the midpoint of the philtrum. The duct exits the superficial part of the parotid gland anteriorly and passes over the masseter muscle prior to entering the oral cavity through the buccinator muscle. The duct can be identified intra-orally via a papilla across from the maxillary second molar. ${ }^{45}$ An injury in the medial third of this line raises concern for an injury of Stensen's duct; injuries laterally can be associated with a direct injury to the parotid gland. Given the association of the buccal branch of the facial nerve to the parotid duct, injuries to the facial nerve should raise concern for a parotid duct injury. Massage of the parotid gland from lateral to medial allows for assessment of salivary fluid exiting the duct or the wound. The majority of saliva produced by the parotid occurs during eating and delayed salivary leak may occur once the patient resumes a diet.

\section{Management}

Cheek lacerations without significant soft tissue loss should be repaired primarily with a layered closure to approximate the mucosa, muscle, and skin. Relative laxity of the skin in the cheek also facilitates primary closure. The vector of tension should run parallel to the margin of the eyelid to avoid causing retraction or ectropion of the lower eyelid. The critical aspects of cheek lacerations are the identification and appropriate management of facial nerve and parotid gland/duct injuries.

Facial nerve transections should be repaired primarily. Early surgical exploration should be performed within 72 hours from the time of injury as this will allow for the use of a nerve stimulator to help identify the distal nerve endings (-Fig. 4). ${ }^{44,46}$ Beyond 72 hours, Wallerian degeneration, or distal nerve demyelination, prevents stimulation of distal nerve endings. If the proximal nerve ends cannot be identified, the facial nerve trunk can be identified either in the parotid gland or in the mastoid bone and followed distally. The nerves should be repaired primarily using standard microsurgical techniques, taking care to avoid tension at the coaptation. If necessary, nerve grafts can be used to bridge gaps and prevent tension. If surgical exploration is not possible within 72 hours, delayed exploration may be considered after reduction of tissue edema and inflammation. Secondary reconstructive procedures such as crossface nerve graft, cranial nerve transfers, temporalis tendon transfer, and free functional muscle transfers can be performed in a delayed fashion. ${ }^{46}$

Parotid duct injuries can be identified by cannulation of the duct papilla. A 22-gauge catheter is inserted into Stensen's duct intra-orally and a small quantity of saline, methylene blue, or Propofol can be injected. ${ }^{45,47}$ If the fluid is seen in the base of the wound, a parotid duct injury can be confirmed. Duct injuries can undergo primary microsurgical repair over a silastic catheter or angiocath. The stent is secured distally and left in place for 10-14 days while the duct heals. ${ }^{47}$ Distal injuries can be diverted into the oral cavity more proximally by passing the distal end of the duct through the buccinator muscle and securing it to the oral mucosa. ${ }^{47}$ Injuries that are too proximal for primary repair or diversion can be managed with ligation of the parotid duct. ${ }^{47}$ This obstruction will eventually atrophy; pressure dressing, antisialogogues such as anticholinergic medications, and/or botulinum toxin can be used to help reduce salivary gland function during this time. Direct parotid gland injuries can be managed with the placement of a drain for 5-7 days in addition to reducing salivary function. Sialocele formation can be managed with serial aspirations, pressure dressings, and antisialogogues.

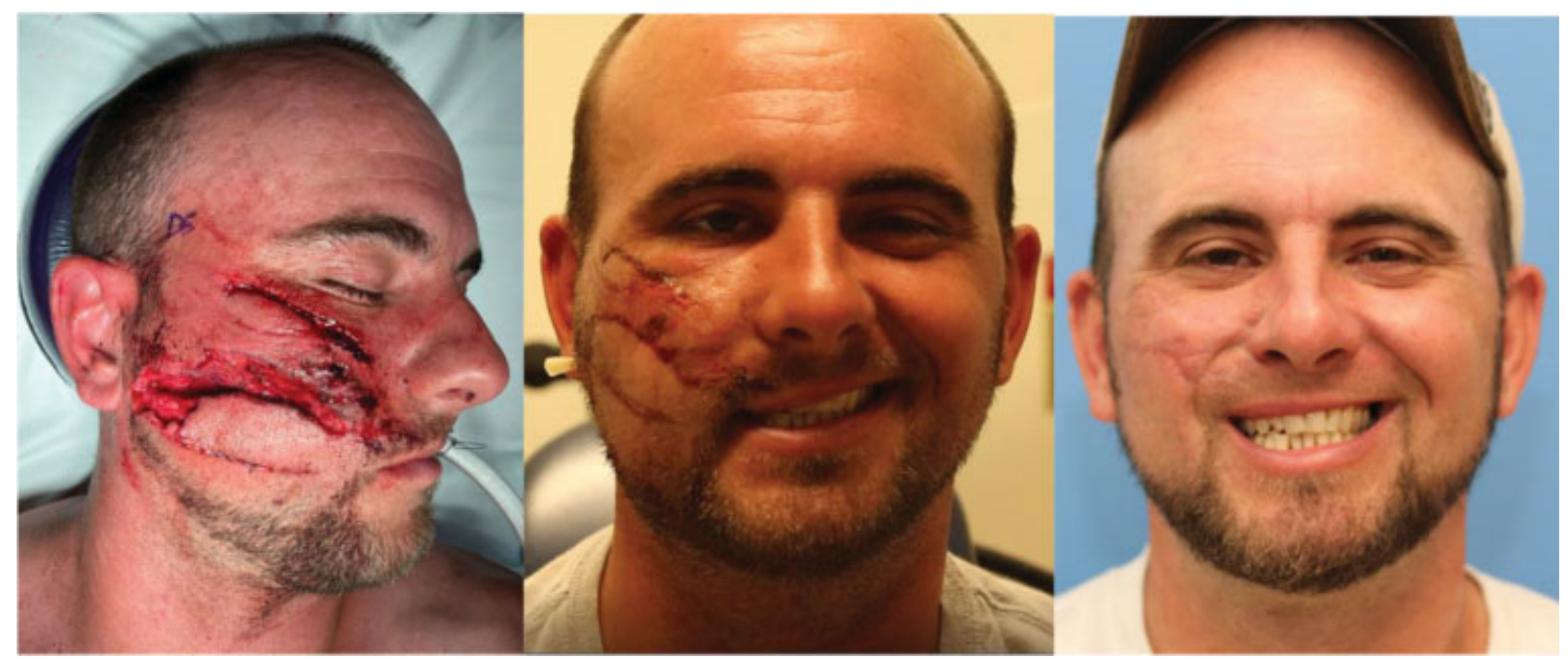

Fig. 4 Initial midface soft tissue injury requiring operative exploration and zygomaticobuccal nerve repair (left), immediate post-operative facial function with right midface weakness (middle), and 8 month post-operative photograph (right) with improvement of facial function. 


\section{Lips}

\section{Evaluation and Special Considerations}

The oral cavity must be carefully examined. The occlusion should be assessed and any loose or missing teeth should be identified; missing teeth must be accounted for or imaging obtained to ensure they have not been aspirated. Evaluating depth and anatomical involvement of lip lacerations is important for restoring form and function of the lips; care must be taken not to miss intra-oral lacerations and injuries. The anatomy of the lips consists of three layers of tissue: mucosa, muscle, and skin. Landmarks include philtrum, cupid's bow, oral commissures, and the vermillion cutaneous border which is the most important landmark. ${ }^{2}$ Retraction of the orbicularis oris muscle often splays open lip injuries making them appear larger than they are.

Marking anatomical landmarks, such as the vermillion, is important prior to injecting local anesthesia that may obscure such landmark. ${ }^{2,48}$ The upper lip receives sensory innervation from the infraorbital nerve, found at the buccal mucosa above the canine tooth. The lower lip receives sensory innervation from the mental nerve, located at the apex of the second premolar. The use of infraorbital blocks and mental blocks will help prevent distortion of the tissue and facilitate anatomic repair. $^{23}$ However, local infiltration of anesthetic may be required in the lateral lip and commissures.

\section{Management}

Goals of repair include maintaining oral competence, optimizing aesthetic outcome, and preventing microstomia. ${ }^{48}$ Repair of full thickness lip lacerations requires the careful approximation of all anatomic structures. Often, a small length of the orbicularis muscle needs to be dissected to facilitate muscle repair; failure to repair the orbicularis can result in a whistle deformity, similar to what is seen in inadequate cleft lip repairs. Once the muscle is approximated, tension is offloaded from the rest of the tissues. This allows for anatomic approximation of the philtrum, cupid's bow, vermillion, and white roll. ${ }^{19}$ Vermillion discrepancies as little as $1 \mathrm{~mm}$ may be visible from speaking distance so care must be taken to be as accurate as possible. Once the critical landmarks are approximated, the skin and the mucosa can be repaired.

Some partial thickness lip injuries can be treated with local wound care and possible secondary scar revision while in other cases, it is favorable to convert a partial thickness injury to a full thickness defect with excision of the remaining tissue. Full thickness defects encompassing less than 30\% of the lip can be closed primarily. ${ }^{20}$ The patient should be warned that their lips might feel tight but will relax over time. Soft tissue loss greater than $30 \%$ of the lip may require local flap reconstruction.

\section{Secondary Reconstruction}

\section{Considerations}

While most soft tissue injuries of the face can be repaired primarily, some may be associated with large defects or tissue loss that require more advanced reconstruction. These injuries often benefit from temporization of the soft tissues with local wound care followed by reconstruction once all the tissue is allowed to declare and the wounds have stabilized especially in the setting of gross contamination, bite injuries, or infection. Many of these injuries are amenable to repair with local and regional flaps or skin grafts but the entire reconstructive ladder should be considered for these injuries from healing by secondary intention ${ }^{42}$ to free flaps and vascularized composite tissue transplantation. ${ }^{49}$

\section{Timing of Revision Procedures}

It is important to counsel patients on the wound healing process at the time of repair. The wound healing process requires 12 months for scars to completely mature. Following any soft tissue injury, it takes 6 weeks for the maximal tensile strength of the scar to develop, which will be $~ 80 \%$ of the tensile strength of the native tissue. ${ }^{50}$ Throughout the course of the scar maturation process, the scar can appear thick and pink due to the collagen deposition and neovascularization process, which slowly settles over time. Non-surgical techniques for scar optimization during this time include minimizing sun exposure, scar massage, silicone gel/sheeting, and steroid injection. ${ }^{51}$ Steroid injection may be considered for hypertrophic scarring starting as early as 6 weeks post injury. Dermabrasion or epidermal resurfacing may be considered starting at 3 months post injury for raised scarring. The timing of formal scar revision should be delayed for 12 to 18 months with serial examinations. Clinical signs that a scar is ready for revision include a maximally mobile and soft scar with an interested and motivated patient.

\section{Conclusions}

Facial soft tissue injuries represent a broad spectrum of presentations, which can often present significant challenges to the craniofacial surgeon. As discussed, a thorough and systematic approach to these patients is critical to ensure that the patient is stabilized, other injuries are not missed, and the full extent of the injuries are properly assessed. Following clear and sound principles, these injuries can be managed to maximize both functional and aesthetic outcomes while minimizing complications.

Conflict of Interest

None declared.

\section{References}

1 Hollander JE, Singer AJ, Valentine S, Henry MC. Wound registry: development and validation. Ann Emerg Med 1995;25(05):675-685

2 Marks M, Polecritti D, Bergman R, Koch CA. Emergent Soft Tissue Repair in Facial Trauma. Facial Plast Surg Clin North Am 2017;25 (04):593-604

3 Perry M. Advanced Trauma Life Support (ATLS) and facial trauma: can one size fit all? Part 1: dilemmas in the management of the multiply injured patient with coexisting facial injuries. Int J Oral Maxillofac Surg 2008;37(03):209-214

4 Perry M, Dancey A, Mireskandari K, Oakley P, Davies S, Cameron $M$. Emergency care in facial trauma-a maxillofacial and ophthalmic perspective. Injury 2005;36(08):875-896 
5 Perry M, Morris C. Advanced trauma life support (ATLS) and facial trauma: can one size fit all? Part 2: ATLS, maxillofacial injuries and airway management dilemmas. Int J Oral Maxillofac Surg 2008;37(04):309-320

6 Perry M, O'Hare J, Porter G. Advanced Trauma Life Support (ATLS) and facial trauma: can one size fit all? Part 3: Hypovolaemia and facial injuries in the multiply injured patient. Int J Oral Maxillofac Surg 2008;37(05):405-414

7 Perry M, Moutray T. Advanced Trauma Life Support (ATLS) and facial trauma: can one size fit all? Part 4: 'can the patient see?' Timely diagnosis, dilemmas and pitfalls in the multiply injured, poorly responsive/unresponsive patient. Int J Oral Maxillofac Surg 2008;37(06):505-514

8 Hopper RA, Salemy S, Sze RW. Diagnosis of midface fractures with CT: what the surgeon needs to know. Radiographics 2006;26(03): 783-793

9 Dreizin D, Nam AJ, Tirada N, et al. Multidetector CT of Mandibular Fractures, Reductions, and Complications: A Clinically Relevant Primer for the Radiologist. Radiographics 2016;36(05):1539-1564

10 Burlew CC, Biffl WL, Moore EE, Barnett CC, Johnson JL, Bensard DD. Blunt cerebrovascular injuries: redefining screening criteria in the era of noninvasive diagnosis. J Trauma Acute Care Surg 2012; 72(02):330-335

11 Franz RW, Willette PA, Wood MJ, Wright ML, Hartman JF. A systematic review and meta-analysis of diagnostic screening criteria for blunt cerebrovascular injuries. J Am Coll Surg 2012; 214(03):313-327

12 Steenburg SD, Sliker CW. Craniofacial gunshot injuries: an unrecognised risk factor for blunt cervical vascular injuries? Eur Radiol 2012;22(09):1837-1843

13 Heckmann N, Simcox T, Kelley D, Marecek GS. Wound Irrigation for Open Fractures. JBJS Rev 2020;8(01):e0061-e0061

14 Heal CF, Banks JL, Lepper PD, Kontopantelis E, van Driel ML. Topical antibiotics for preventing surgical site infection in wounds healing by primary intention. Cochrane Database Syst Rev 2016;11:CD011426

15 Jenzer AC, Storrs BP, Daniels Z, Hanlon JJ. Traumatic Facial Tattoo Injuries From Gunpowder and Ammunition: A Case Series. Craniomaxillofac Trauma Reconstr 2020;13(02):133-137

16 Bernstein S, Barros B, Zaenglein AL. A Gunpowder Tattoo in a 6Year-Old Girl. Pediatr Dermatol 2016;33(03):e210-e211

17 Powers DB, Breeze J. Avulsive Soft Tissue Injuries. Atlas Oral Maxillofac Surg Clin North Am 2019;27(02):135-142

18 Vaca EE, Bellamy JL, Sinno S, Rodriguez ED. Management of Highenergy Avulsive Ballistic Facial Injury: A Review of the Literature and Algorithmic Approach. Plast Reconstr Surg Glob Open 2018;6 (03):e1693

19 Sabatino F, Moskovitz JB. Facial wound management. Emerg Med Clin North Am 2013;31(02):529-538

20 Braun TL, Maricevich RS. Soft Tissue Management in Facial Trauma. Semin Plast Surg 2017;31(02):73-79

21 Jin Y, Hua C, Hu X, et al. Microsurgical Replantation of Total Avulsed Scalp: Extending the Limits. J Craniofac Surg 2017;28 (03):670-674

22 PITANGUY IRAMOS AS. The Frontal Branch of the Facial Nerve. Plast Reconstr Surg 1966;38(04):352-356

23 Zide BM, Swift R. How to block and tackle the face. Plast Reconstr Surg 1998;101(03):840-851

24 Frank SG, Lalonde DH. How acidic is the lidocaine we are injecting, and how much bicarbonate should we add? Can J Plast Surg 2012; 20(02):71-73

25 Hicks DL, Watson D. Soft tissue reconstruction of the forehead and temple. Facial Plast Surg Clin North Am 2005;13(02):243-251, vi

26 Soparkar CNS, Patrinely JR. The eye examination in facial trauma for the plastic surgeon. Plast Reconstr Surg 2007;120(07, Suppl 2):49S-56S
27 Beadles KA, Lessner AM. Management of traumatic eyelid lacerations. Semin Ophthalmol 1994;9(03):145-151

28 MacGillivray RF, Stevens MR. Primary surgical repair of traumatic lacerations of the lacrimal canaliculi. Oral Surg Oral Med Oral Pathol Oral Radiol Endod 1996;81(02):157-163

29 Chang EI, Esmaeli B, Butler CE. Eyelid Reconstruction. Plast Reconstr Surg 2017;140(05):724e-735e

30 Liston SL, Cortez EA, McNabney WK. External ear injuries. JACEP 1978;7(06):233-236

31 Tubbs RS, Shoja MM, Loukas M, Oakes WJ, Cohen-Gadol A. William Henry Battle and Battle's sign: mastoid ecchymosis as an indicator of basilar skull fracture. J Neurosurg 2010;112(01): 186-188

32 Greywoode JD, Pribitkin EA, Krein H. Management of auricular hematoma and the cauliflower ear. Facial Plast Surg 2010;26(06): 451-455

33 Salam GA. Regional anesthesia for office procedures: part I. Head and neck surgeries. Am Fam Physician 2004;69(03):585-590

34 Elsahy NI. Acquired ear defects. Clin Plast Surg 2002;29(02): 175-186, v-vi

35 Templer J, Renner GJ. Injuries of the external ear. Otolaryngol Clin North Am 1990;23(05):1003-1018

36 Elsahy NI. Ear replantation. Clin Plast Surg 2002;29(02):221-231, vi-vii

37 Sullivan SR, Taylor HO. Images in clinical medicine. Ear replantation. N Engl J Med 2014;370(16):1541-1541

38 Sanyaolu LN, Farmer SEJ, Cuddihy PJ. Nasal septal haematoma. BMJ 2014;349:g6075

39 Dar PMUD, Gupta P, Kaul RP, et al. Haemorrhage control beyond Advanced Trauma Life Support (ATLS) protocol in life threatening maxillofacial trauma - experience from a level I trauma centre. $\mathrm{Br} \mathrm{J}$ Oral Maxillofac Surg 2021;59(06):700-704

40 Kucik CJ, Clenney T. Management of epistaxis. Am Fam Physician 2005;71(02):305-311

41 Thiagarajan B. Local Anaesthesia of Nose and Nasal cavity-A Review. Global J Otolaryngology 2017;4(04):88-90

42 Goldwyn RM, Rueckert F. The value of healing by secondary intention for sizeable defects of the face. Arch Surg 1977;112 (03):285-292

43 Green Sanderson K, Conti A, Colussi M, Connolly C. A Simple Clinical Application for Locating the Frontotemporal Branch of the Facial Nerve Using the Zygomatic Arch and the Tragus. Aesthet Surg J 2020;40(05):NP223-NP227

44 Davis RE, Telischi FF. Traumatic facial nerve injuries: review of diagnosis and treatment. J Craniomaxillofac Trauma 1995;1(03): 30-41

45 Demian N, Curtis W. A simple technique for cannulation of the parotid duct. J Oral Maxillofac Surg 2008;66(07):1532-1533

46 Gosain AK, Matloub HS. Surgical management of the facial nerve in craniofacial trauma and long-standing facial paralysis: cadaver study and clinical presentations. J Craniomaxillofac Trauma 1999; 5(01):29-37

47 Steinberg MJ, Herréra AF. Management of parotid duct injuries. Oral Surg Oral Med Oral Pathol Oral Radiol Endod 2005;99(02): 136-141

48 Houle A, Markiewicz MR, Callahan N. Soft Tissue Trauma Management of Lip Injury. Oral Maxil Surg Clin 2021

49 Losee JE, Fletcher DR, Gorantla VS. Human facial allotransplantation: patient selection and pertinent considerations. J Craniofac Surg 2012;23(01):260-264

50 Levenson SM, Geever EF, Crowley LV, Oates JF III, Berard CW, Rosen H. The Healing of Rat Skin Wounds. Ann Surg 1965; 161:293-308

51 Mustoe TA, Cooter RD, Gold MH, et al; International Advisory Panel on Scar Management. International clinical recommendations on scar management. Plast Reconstr Surg 2002;110(02):560-571 